\title{
Qualidade de sono em universitários de uma instituição privada
}

\section{Sleep quality in universities in a private institution}

\section{Cleane Ribeiro de Oliveira' ${ }^{1}$ Brena Costa de Oliveira ${ }^{2}$ (1) Luana Gabrielle França Ferreira ${ }^{3}$ (1)}

Centro Universitário UNINASSAU (Teresina). Piauí, Brasil. cleaneribeiro.18@gmail.com ²Universidade Federal do Piauí (Teresina).Piauí, Brasil. brena_oliveira.5@hotmail.com ${ }^{3}$ Autora para correspondência. Universidade Federal do Piauí (Teresina), Centro Universitário UNINASSAU (Teresina). Piauí, Brasil. luanagabrielle@yahoo.com.br

\begin{abstract}
RESUMO | INTRODUÇÃO: O sono é uma suspensão normal e temporária do nível de consciência, fazendo parte do ciclo sono vigília, sendo regulado por um componente circadiano. Os universitários estão propensos a obter distúrbios do sono devido ao estilo de vida e fatores ambientais. OBJETIVO: Investigar a qualidade de sono, presença de sonolência diurna excessiva e fatores associados em estudantes do curso de fisioterapia de uma faculdade privada. METODOLOGIA: Tratase de um estudo de campo de caráter transversal, quantitativo onde os voluntários foram avaliados através do Índice de Qualidade de Sono de Pittsburgh (PSQI) e escala de sonolência de Epworth. Foram incluídos alunos do curso de fisioterapia e excluídos aqueles não preencheram completamente os instrumentos e aqueles que tomaram medicamentos que afetam o sono. Para a análise estatística, foi utilizado um intervalo de confiança de $95 \%$ e nível de significância de $5 \%$ ( $p \leq 0,05)$. RESULTADOS: Foram 80 estudantes, $71,3 \%$ eram do sexo feminino, $80 \%$ eram solteiros e do turno da manhã, $63,5 \%$ não trabalhavam, 46,3\% praticavam atividade física. Quanto à qualidade de sono, observou-se média do escore do PSQI de 9,5 $\pm 3,6$, com predomínio de má qualidade de sono nos estudantes com $82,5 \%$. Em relação à presença de sonolência diurna excessiva (SED) nos estudantes observou-se média de escore da escala de Epworth de 11,2 $\pm 4,5$, com $55 \%$ dos estudantes apresentando SED. Não houve diferença entre os escores de PSQI e Epworth entre os períodos do curso, turno de estudo e estudantes trabalhadores. CONCLUSÃO: Houve maior prevalência de alunos com sono ruim, onde a sonolência diurna excessiva foi destacada na maioria. A má qualidade de sono atingiu aos estudantes sem diferenciação entre períodos do curso, turno de estudo e trabalho.
\end{abstract}

PALAVRAS-CHAVE: Estudantes. Sono. Saúde.

\begin{abstract}
INTRODUCTION: Sleep is a normal and temporary suspension of the level of consciousness, being part of the sleep-wake cycle, being regulated by a circadian component. College students are likely to get sleep disorders due to lifestyle and environmental factors. OBJECTIVE: To investigate the quality of sleep, the presence of excessive daytime sleepiness and associated factors in students of the physiotherapy course at a private college. METHODOLOGY: This is a cross-sectional, quantitative field study where volunteers were assessed using the Pittsburgh Sleep Quality Index (PSQI) and Epworth sleepiness scale. Physiotherapy students were included and those who did not completely fill out the instruments and those who took medications that affect sleep were excluded. For the statistical analysis, a $95 \%$ confidence interval and a significance level of 5\% ( $p$ $\leq 0.05$ ) were used. RESULTS: There were 80 students, $71.3 \%$ were female, $80 \%$ were single and in the morning shift, $63.5 \%$ did not work, $46.3 \%$ practiced physical activity. As for sleep quality, a mean PSQI score of $9.5 \pm 3.6$ was observed, with a predominance of poor sleep quality in students with $82.5 \%$. Regarding the presence of excessive daytime sleepiness (SED) in students, an average Epworth scale score of $11.2 \pm 4.5$ was observed, with $55 \%$ of students presenting EDS. There was no difference between the PSQI and Epworth scores between the periods of the course, study shift and working students. CONCLUSION: There was a higher prevalence of students with poor sleep, where excessive daytime sleepiness was highlighted in the majority. The poor quality of sleep reached students without differentiating between periods of the course, study shift and work.
\end{abstract}

KEYWORDS: Students. Sleep. Health. 


\section{Introdução}

O sono pode ser definido como suspensão normal e temporária do nível de consciência, faz parte do ciclo vigília - sono, e é regulado por um componente circadiano determinado pelo relógio biológico e por um processo homeostático que é caracterizado pela pressão do mesmo'. É composto por duas fases importantes, a fase dos movimentos rápidos dos olhos (REM) e a fase sem movimentos rápidos dos olhos (NREM), representando distintos componentes neurais. O sono exerce influência nas funções endócrinas, termorregulação, conservação de energia, tem uma função biológica fundamental na consolidação da memória².

Destacando o sono em universitários, sabe-se que esses estão propensos a obter distúrbios do sono, pois a rotina de vida e fatores ambientais pode levar a essas ocorrências, as necessidades impostas pela sociedade como horário de aulas e trabalho podem acarretar em uma má qualidade do sono e assim consequentemente redução no rendimento acadêmico e profissional $^{3}$.

Esta população que em sua maioria jovem, pode apresentar maior privação de sono com tendência a sonolência diurna excessiva, tem como consequência, problemas de memória, diminuição do rendimento acadêmico, irritabilidade, problemas comportamentais como estresse e ansiedade ${ }^{4}$.

Além dos estudos, os universitários que trabalham podem ter esse fator como um agravante da má qualidade do sono, que poderá impactar na má produtividade no trabalho e um rendimento ruim nos estudos $^{5}$. A privação do sono inclui um mau desempenho cognitivo, não restauração da memória, resultando também em estados de humor negativo, sentimento de fadiga, sonolência durante as atividades diárias e confusão mental ${ }^{6}$.

Com o intuito de atender cobranças no meio acadêmico, estudantes universitários adequam seu sono de acordo com as atividades curriculares, tornando seus horários de sono irregulares e não reparadores para o corpo ${ }^{6}$. Neste contexto, objetiva-se investigar a qualidade de sono, a presença de sonolência diurna excessiva e fatores associados em estudantes do curso de fisioterapia de uma faculdade privada.

\section{Metodologia}

Trata-se de um estudo de campo de caráter transversal e quantitativo, os estudantes universitários foram avaliados quanto à qualidade do sono. $O$ estudo foi realizado em uma Faculdade de Teresina-PI, no período de outubro e novembro de 2019 , com a população de 255 alunos matriculados no curso de Fisioterapia. A amostra foi por conveniência aleatória simples. Os critérios de inclusão foram alunos matriculados no curso e que aceitaram participar do estudo. Foram excluídos os estudantes que não preencheram completamente os instrumentos de coletas de dados e alunos que relataram fazer uso de medicamentos que afetam o sono, como hipnóticos e antidepressivos.

A coleta de dados foi por meio do questionário de Índice de Qualidade de Sono de Pittsburgh (PSQI), desenvolvido por Buysse et al. (1989) ${ }^{7}$ e validado no Brasil, em população adulta, por Bertolazi et al. $(2011)^{8}$. O PSQI é um instrumento que foi criado para avaliar a qualidade de sono em relação ao último mês, composto por 24 perguntas, quatro subjetivas e vinte objetivas. Os sete domínios nesse questionário são: 1-Qualidade subjetiva do sono; 2- latência de sono; 3- Duração do sono; 4- Eficiência habitual do sono; 5- Distúrbios do sono; 6- Uso de medicamentos; 7- Sonolência diurna e os distúrbios durante o dia. O PSQI apresenta escore final variando entre $0 \mathrm{e}$ 21. A partir do escore final é possível classificar o indivíduo em boa $(<6)$ ou ruim $(>5)$ qualidade do sono ${ }^{8}$.

O outro instrumento foi a escala de sonolência de Epworth $^{9}$, utilizada para avaliar sonolência diurna. Consiste em oito questões que descrevem situações diárias que podem induzir à sonolência. Cada questão é graduada de 0 a 3 pontos podendo chegar a escore total de 24 . 0 escore $>10$ classifica o indivíduo com sonolência diurna excessiva9. As informações obtidas através do questionário nas questões abertas foram classificadas e agrupadas para uma melhor análise de dados, o tempo de aplicação dos instrumentos durou em média 30 minutos. 
Os dados foram organizados em planilha no programa Microsoft Excel versão 8.0 e posteriormente exportados para o programa Statistical Package for the Social Sciences (SPSS) versão 22.0, sendo as variáveis descritas por meio de porcentagem, média, mediana e desvio padrão. A análise dos dados categóricos tais como sexo e estado civil foi feita pela medida de associação Qui-quadrado e apresentação em porcentagens (descritiva). Para análise das variáveis contínuas (como idade) foi realizada a verificação da normalidade dos dados pelo teste Kolmogorov-Smirnov para posteriormente determinação dos testes de análise comparativa (Teste t). Foi considerado um intervalo de confiança de $95 \%$ e nível de significância de $5 \%(p \leq 0,05)$.

A coleta de dados ocorreu após a aprovação pelo Comitê de Ética em Pesquisa (CEP) com parecer n 3.501 .694 (CAAE 15768619.7.0000.5210). Os voluntários foram esclarecidos e autorizaram a participação na pesquisa por meio do Termo de Consentimento Livre e Esclarecido (TCLE).

\section{Resultados}

Participaram da pesquisa 80 estudantes, sendo 71,3\% (57) do sexo feminino, 80\% (64) estudantes eram solteiros e do turno manhã, 67,5\% (54) não trabalham, 46,3\% (37) praticam atividade física, como mostra a Tabela 1.

Tabela 1. Caracterização sociodemográfica dos estudantes de fisioterapia participantes da pesquisa. Teresina-PI, 2019

\begin{tabular}{lc}
\hline Variáveis & $\mathbf{N}(\%)$ \\
\hline Sexo & $57(71,3 \%)$ \\
Feminino & $23(28,7 \%)$ \\
Masculino & \\
\hline Estado civil & $65(81,2 \%)$ \\
Solteiro & $15(18,8 \%)$ \\
Casado & \\
\hline Trabalho & $26(32,5 \%)$ \\
Sim & $54(67,5 \%)$ \\
Não & \\
\hline Turno de estudo & $64(80 \%)$ \\
Manhã & $16(20 \%)$ \\
Noite & \\
\hline Período & $20(25 \%)$ \\
$4^{\circ}$ & $26(32,5 \%)$ \\
$6^{\circ}$ & $16(20 \%)$ \\
$8^{\circ}$ & $18(22,5 \%)$ \\
$10^{\circ}$ & $38(47,5 \%)$ \\
\hline Atividade física & $42(52,5 \%)$ \\
Sim & \\
Não & \\
\hline
\end{tabular}

Fonte: Dados da pesquisa.

Com relação à avaliação da qualidade de sono, observou-se média do escore do PSQI de 9,5 $\pm 3,6$, com predomínio de má qualidade de sono nos estudantes investigados com 82,5\%, Figura 1. 
Figura 1. Caracterização da qualidade de sono dos estudantes de fisioterapia. Teresina-PI, 2019

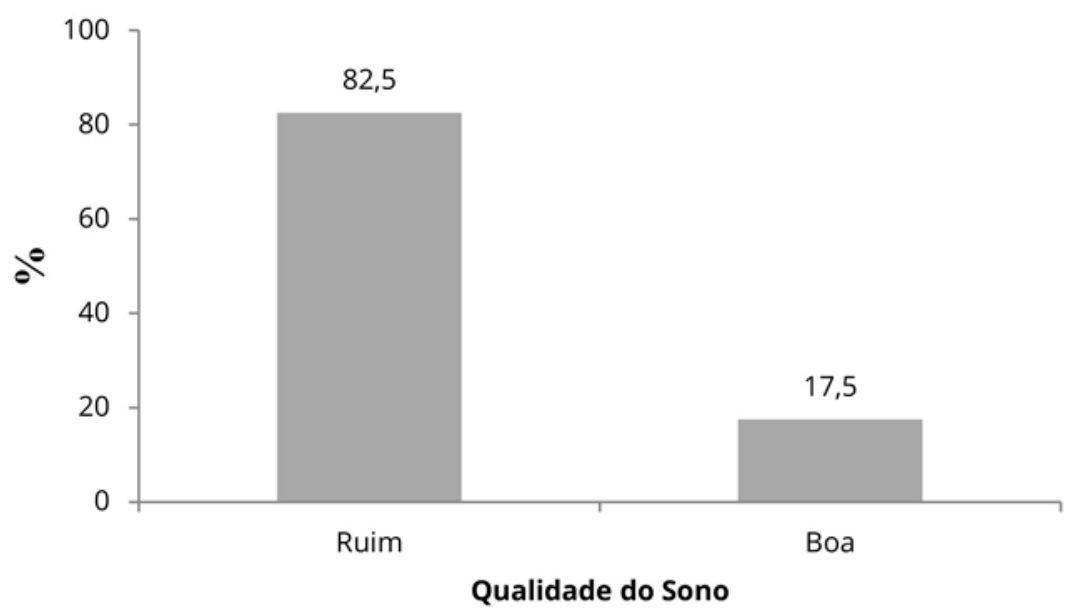

Fonte: Dados da pesquisa.

Por meio do instrumento PSQI, pode-se ainda caracterizar os domínios que compõe a qualidade do sono, observando-se os domínios "duração do sono" e "eficiência do sono" com maiores médias contribuindo com o escore final do PSQI.

Tabela 2. Domínios da qualidade do sono dos estudantes de fisioterapia. Teresina-PI, 2019

\begin{tabular}{ll}
\hline Domínios do PSQI & Média (DP) \\
\hline C1 - qualidade do sono & $1,4 \pm 0,8$ \\
\hline C2 - latência do sono & $1,4 \pm 0,8$ \\
\hline C3 - duração do sono noturno & $1,7 \pm 1,1$ \\
\hline C4 - eficiência do sono & $1,7 \pm 1,4$ \\
\hline C5 - distúrbio do sono noturno & $1,6 \pm 0,6$ \\
\hline C6 - medicação para dormir & $0,3 \pm 0,7$ \\
\hline C7 - sonolência e distúrbios do sono & $1,4 \pm 0,9$ \\
\hline Escore do PSQI & $9,5 \pm 3,6$ \\
\hline
\end{tabular}

Fonte: Dados da pesquisa.

Investigando a presença de sonolência diurna excessiva (SED) nos estudantes, observou-se média de escore da escala de Epworth de 11,2 $\pm 4,5$, com 55\% (44) dos estudantes apresentando SED. 


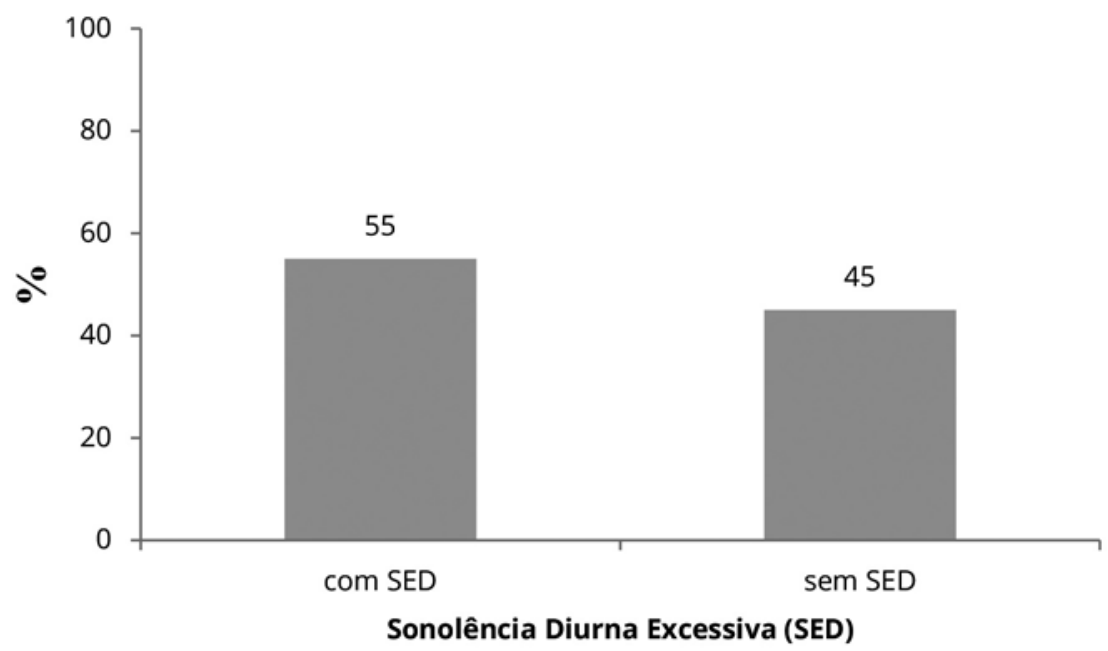

Fonte: Dados da pesquisa.

Destaca-se que não houve diferença estatística $(p>0,05)$ entre os escores de qualidade de sono (manhã $=9,8$ $\pm 3,5$ versus noturno $=8,3 \pm 4,2 ; p=0,17$ ) e escores de sonolência diurna excessiva (manhã $=11,5 \pm 4,8$ versus noturno $=9,9 \pm 3,2 ; p=0,21$ ) quando se comparou os turnos de estudo dos universitários. Também não houve diferença $(p>5)$ entre escores de PSQI e Epworth entre períodos acadêmicos e estudantes trabalhadores. As figuras 3 e 4 abaixo mostram a comparação de qualidade do sono e SED entre os turnos.

Figura 3. Comparação do escore PSQI dos estudantes entre os turnos de estudo. Teresina-PI, 2019

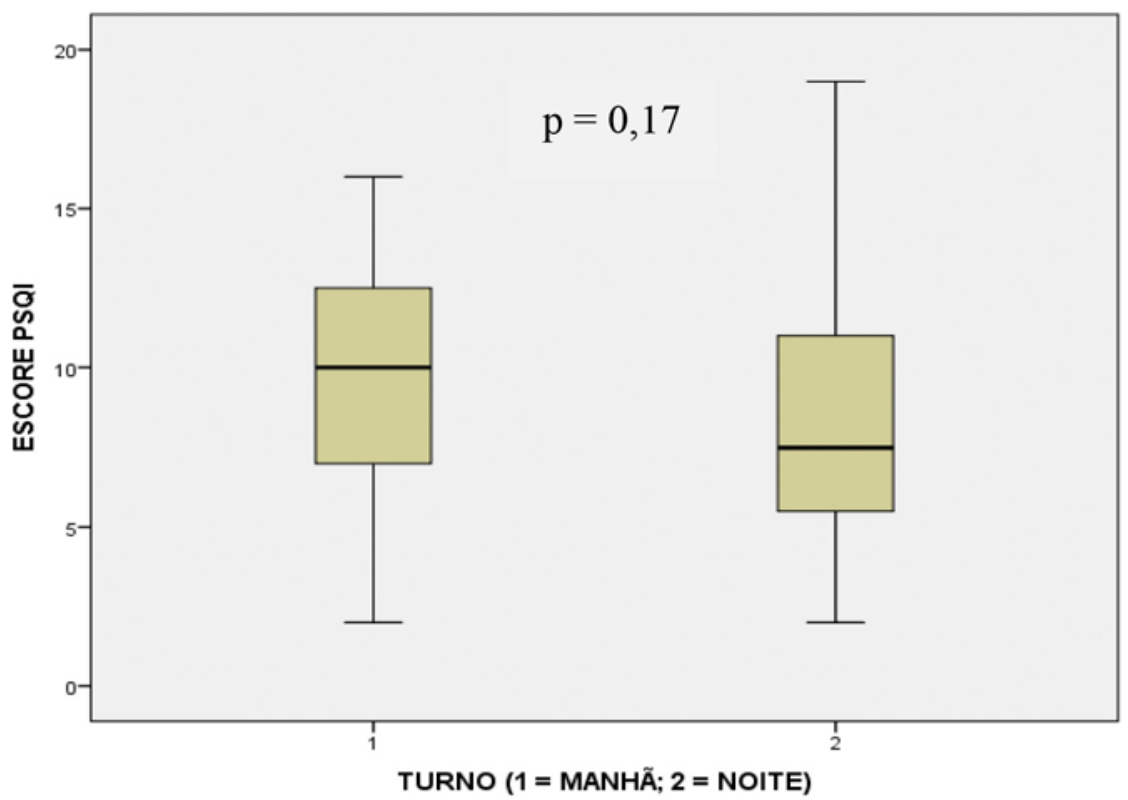




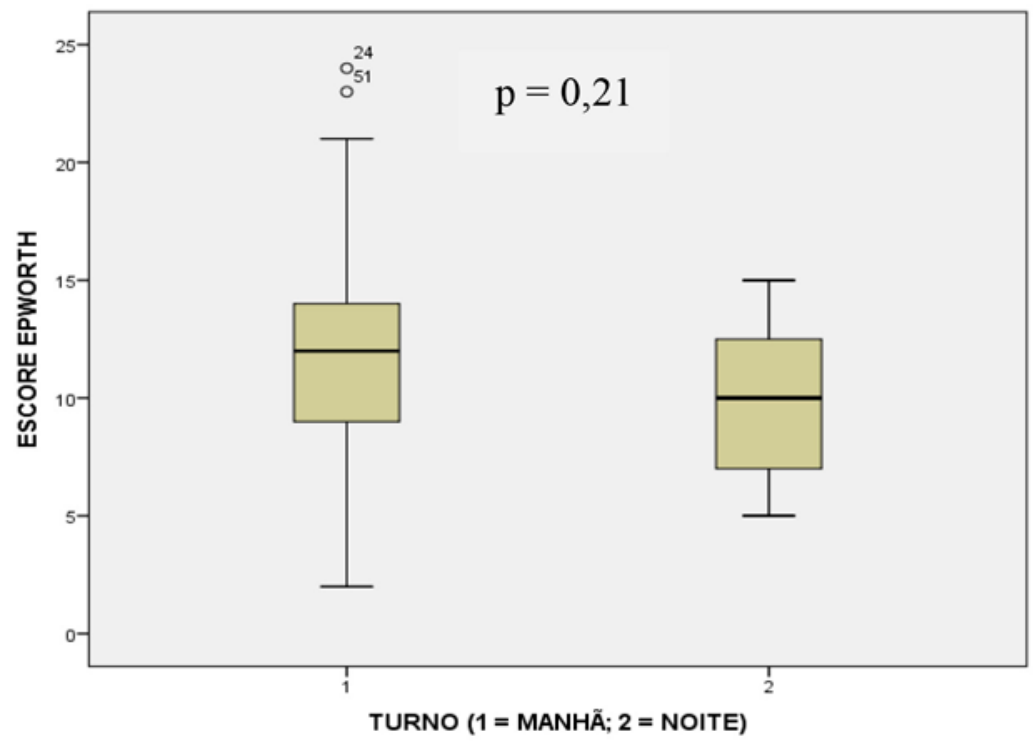

\section{Discussão}

Este estudo revelou que há uma alta frequência de estudantes de fisioterapia com má qualidade do sono e de sonolência diurna excessiva. Observou-se ainda que os domínios "duração do sono" e "eficiência do sono" impactaram no escore de qualidade do sono. Nesta pesquisa não se observou associação da qualidade do sono com turno de estudo, período acadêmico e trabalho.

A prevalência de má qualidade do sono encontrada neste estudo foi maior do que a encontrada em uma pesquisa feita com os estudantes de enfermagem onde $67,0 \%$ apresentaram uma má qualidade do sono ${ }^{10}$. Em outro estudo ${ }^{11}$ com estudantes de medicina do sul do Brasil, houve uma média de PSQI de 7,2 $\pm 3,0$ e $70,6 \%$ dos estudantes referiram má qualidade do sono. Entretanto, o tamanho amostral desse estudo foi 143 estudantes de medicina, em média dormiam $6,0( \pm 1,1)$ horas, latência de $21,3( \pm 17,5)$ minutos e eficiência do sono de $91,9 \%( \pm 11,9)$, resultados menores que o apresentado nesta presente pesquisa. A pesquisa com estudantes dos primeiros períodos foi realizada com os primeiros períodos de curso diurno, o que difere do perfil da presente pesquisa. Com relação ao semestre do curso não teve resultados significantes para uma alteração entre eles, corroborando com a presente pesquisa.
Sobre os domínios que impactaram na qualidade do sono, o estudo feito com os estudantes de medicina relatou que o horário de deitar e a latência do sono foram significativamente maiores no grupo com má qualidade de sono em relação ao grupo com boa qualidade de sono. Além disso, os domínios duração e a eficiência do sono foram significativamente menores no grupo com má qualidade de sono ${ }^{11}$, corroborando com a presente pesquisa. Um aspecto relevante é que a pesquisa, com estudantes de medicina, foi realizada no início do semestre letivo, mostrando que ainda não estavam sendo expostos a cargas rigorosas de estudos, concluído assim que estudantes possuem hábitos de sono ruim onde dormir tarde e ficar expostas a TV e celular durante a noite atrapalha a rotina de sono saudável ${ }^{11}$.

Em relação à diferença de escore de qualidade de sono e sonolência diurna excessiva quando comparado os turnos de estudo, os períodos dos acadêmicos e os estudantes trabalhadores não houve diferença entre os participantes do estudo. No entanto, no estudo no Paraná-Brasil com estudantes de fisioterapia com má qualidade do sono apresentaram maior prevalência de SDE e os mesmos trabalhavam em um ou dois turnos ${ }^{4}$. 
Em outro estudo também realizado em uma universidade privada com estudantes de fisioterapia mostrou que estudantes do último período acadêmico apresentaram $66 \%$ de SED em relação aos dos primeiros períodos que foi de $32,6 \%$. Essa diferença foi obtida provavelmente pela grande demanda de afazeres empregada pelos acadêmicos do último período ${ }^{12}$. Já na presente pesquisa destaca-se que não houve diferença estatística entre os escores de qualidade de sono e escores de sonolência diurna excessiva quando se comparou os turnos de estudo.

Uma das causas da SED é a curta duração de sono, e essa privação do sono em grande parte de universitários impacta na percepção saúde menos positiva do que aqueles que apresentam uma taxa de sono maior. Andrade et al. (2017) ${ }^{13}$ apontou em seu estudo que quanto mais dias de sono insuficientes, maior é a percepção negativa de saúde.

Destaca-se que intervenções podem ser implantadas pelas instituições de ensino com ações para melhorar ou minimizar efeitos que estão ligados à privação do sono. Como exemplo, os programas de higienização e conscientização de hábitos do sono, podem contribuir, onde os universitários podem estar cientes da importância de dormir bem e ter a autonomia na adesão de hábitos saudáveis ${ }^{4}$.

As limitações deste estudo foram referentes à subjetividade dos instrumentos utilizados e a impossibilidade de detectar distúrbios do sono que explicassem a má qualidade do sono a partir dos métodos do estudo que foram avaliados a partir do questionário autoaplicável, o que pode levar a subestimação dos problemas apresentados, com possibilidade de erros de preenchimento ou viés de memória. É essencial que outros estudos sejam feitos com intervenções, como higiene do sono e estímulo a hábitos saudáveis sejam testadas em busca de uma melhor qualidade do sono.

\section{Conclusão}

Esse estudo aponta uma maior frequência de universitários com má qualidade de sono e sonolência diurna excessiva. Não houve associação entre a qualidade de sono e a sonolência diurna com turno de estudo, trabalho ou período acadêmico demostrando que a má qualidade de sono atinge os alunos de forma geral.

\section{Contribuições dos autores}

Oliveira CR participou da concepção, delineamento, busca, coleta de dados, tabulação, interpretação dos resultados, redação do artigo científico. Ferreira LGF participou da concepção, delineamento, busca e análise estatística dos dados da pesquisa, interpretação dos resultados, redação do artigo científico. oliveira BC participou da interpretação dos dados e redação do artigo científico.

\section{Conflitos de interesses}

Nenhum conflito financeiro, legal o político envolvendo terceiros (gobierno, empresas y fundaciones privadas, etc.) foi declarado para nenhum aspecto do trabalho submetido (incluido más não limitando-se a subvenções e financiamentos, participación en conselho consultivo, desenho de estudo, preparación de manuscrito, análise estatística, etc.).

\section{Referências}

1. Crabtree VMcL, Williams NA. Normal sleep in children and adolescents. Child Adolesc Psychiatr Clin N Am. 2009;18(4):799811. doi: 10.1016/j.chc.2009.04.013

2. Mello BJ, Mello ST, Vidotti AP, Mello JM. Cronotipo e qualidade do sono de acadêmicos do primeiro ano do curso de medicina da cidade de Maringá-PR. Revista Saúde e Pesquisa. 2018;11(2):287292. doi: $10.17765 / 2176-9206.2018 v 11 n 2 p 287-292$

3. Al-Kandari S, Alsalem A, Al-Mutairi S, Al-Lumai D, Dawoud A, Moussa M. Association between sleep hygiene awareness and practice with sleep quality among Kuwait University students. Sleep Health. 2017;3(5):342-347. doi: 10.1016/j.sleh.2017.06.004

4. Martini M, Brandalize M, Louzada FM, Pereira EF, Brandalize D. Fatores associados à qualidade do sono em estudantes de Fisioterapia. Fisioter Pesq. 2012;19(3):261-267. doi: $10.1590 /$ \$1809-29502012000300012 
5. Ferreira LRC, Martino MMF. Padrão de sono e sonolência do trabalhador estudante de enfermagem. Rev Esc Enferm USP. 2012;46(5):1178-1183. doi: 10.1590/S0080-62342012000500020

6. Carvalho TMCS, Silva Junior II, Siqueira PPS, Almeida JO, Soares AF, Lima AMJ. Qualidade do sono e sonolência diurna entre estudantes universitários de diferentes áreas. Rev Neurociênc. 2013;21(3):383-387. doi: 10.4181/RNC.2013.21.854.5p

7. Buysse DJ, Reynolds CF, Monk TH, Berman SR, Kupfer DJ. The Pittsburgh Sleep Quality Index: a new instrument for psychiatric practice and research. Psychiatry Res. 1989;28(2):193-213. doi: 10.1016/0165-1781(89)90047-4

8. Bertolazi AN, Fagondes SC, Hoff LS, Dartora EG, Miozzo ICS, Barba MEF et al. Validation of the Brazilian Portuguese version of the Pittsburgh Sleep Quality Index. Sleep Med. 2011;12(01):70-5. doi: $10.1016 /$ j.sleep.2010.04.020

9. Bertolazi NA, Fagondes SC, Hoff LS, Pedro VD, Barreto SSM, Johns MW. Validação da escala de sonolência de Epworth em português para uso no Brasil. J Bras Pneumol. 2009;35(09):877-83. doi: 10.1590/S1806-37132009000900009

10. Coelho AFM. Avaliação da qualidade do sono em estudantes universitários e a sua relação com disfunções temporomandibulares musculares [dissertação]. Porto: Universidade Fernando Pessoa; 2014.

11. Ferreira CMG, Kluthcovsky ACGC, Dornelles CF, Stumpf MAM, Cordeiro TMG. Qualidade do sono em estudantes de medicina de uma universidade do Sul do Brasil. Conexão Ci. 2017;12(2):78-85. doi: $10.24862 /$ cco.v12i1.501

12. Moraes JB, Siebra TS, Paz KRA, Luz Filho CA, Luz KRG, Sousa $A C$ et al. Sonolência excessiva diurna em alunos do último ano do curso de fisioterapia de uma faculdade privada. Revista Eletrônica Acervo Saúde. 2019;(17):e371. doi: 10.25248/reas.e371.2019

13. Andrade RD, Felden EPG, Teixeira CS, Pelegrini A. Sono, percepção de saúde e atividade física em adolescentes universitários. Adolesc. Saúde. 2017;14(4):150-156.

14. Lopes HS, Meier DAP, Rodrigues R. Qualidade do sono entre estudantes de enfermagem e fatores associados. Semina: Ciências Biológicas e da Saúde. 2018;39(2):129-136. doi:

10.5433/1679-0367.2018v39n2p129 